\title{
Homology: Why We Know a Whale Is Not a Fish
}

\author{
Andrew J. Petto • Louise S. Mead
}

Published online: 20 October 2009

(C) Springer Science + Business Media, LLC 2009

\begin{abstract}
Homology is a fundamental concept in comparative and evolutionary biology and yet often the focus of antievolution challenges. In describing structural similarity that is the result of common ancestry, hypotheses about homology require rigorous testing and form the basis for making predictions about anatomy and physiology as well as the fossil record. Communicating the basics of homology to students is essential for a high school biology curriculum.
\end{abstract}

Keywords Classification · Evolution · Homology

\section{Introduction}

Biological classification is a mainstay of $\mathrm{K}-12$ life science curricula. When we understand, for example, that a whale is a mammal and not a fish, we immediately know a tremendous amount about its biology: its reproductive, circulatory, and nervous systems; its physiological temperature regulation; its muscles, skin, bones, and so on. This knowledge is derived from the fact that all mammals share a number of derived biological characteristics that demonstrate their common ancestry from a specific branch of vertebrates.

This evolutionary classification is not the only way that humans classify organisms, of course. The anthropological field known as "ethnobiology" investigates the various

A. J. Petto

Department of Biological Sciences, University of Wisconsin,

P.O. Box 413, Milwaukee, WI 53201-0413, USA

A. J. Petto $\cdot$ L. S. Mead $(\bowtie)$

National Center for Science Education,

P.O. Box 9477, Berkeley, CA 94709-0477, USA

e-mail: mead@ncseweb.org ways that organisms are classified in different cultures, and these "folk" categories often do bear a reasonably close relationship to formal taxonomic categories (for example, Begossi et al., 2008) when they rely primarily on observations from nature. Other folk systems do exist, however, that produce classifications that are very different from modern biology (for example, Berndt 2000; see review by Petto and Meyers 2004).

Perhaps it is this close correspondence of folk and formal scientific classifications that underlies some of the misconceptions about biological classification and explains why antievolution arguments often include attacks on homology, effectively exploiting the public's confusion between scientific and folk systems of classification. In the Biblical Classification of Life (2000), Berndt's mischaracterization of the "Modern Taxonomy" as grouping "life on the basis of physical similarity" (p. 23) reiterates two common misconceptions - that taxonomy is synonymous with classification and that modern biological classification is based on physical similarity. Nickels and Nelson (2005) illustrate the potential problems with the folk model for classification - "like goes with like" - arguing cogently that students should learn that the basis for biological classification is phylogeny, emphasizing that it is neither overall similarity nor even the number of similarities that matters; rather, it is the subset of similarities that are homologous, and more specifically those character states that are uniquely shared by the ancestor and all descendants, that we use to construct hypotheses about evolutionary relationships.

\section{Understanding Homology}

The fundamental concept of evolutionary homology is not difficult to describe: homologies are those features that are 
similar in structure and position in two or more organisms because these features existed in and were inherited from a common ancestor (for additional discussion of homology see Thanukos 2008). A number of features of the concept as described here demand attention. First, not all similarities count as homologies: only those similarities owing to common descent do. There are many examples for which overall structural similarity results from functional or environmental constraints rather than shared ancestry: webbing between digits in aquatic organisms, crosssectional proportions of wings in flying animals, modifications of leaves in cold and/or dry environments. Alternative hypotheses to explain structural similarity, such as convergence, do not undermine common ancestry as a powerful explanation.

Second, the concept of homology is only a definition, not a diagnosis: on its own, it provides no guidance about how to tell whether features are homologous. Unfortunately, many textbooks, in simplifying content, confuse the definition of homology with the diagnosis of a homologous structure (Gishlick 2006), a confusion that is often repeated in criticisms of evolution (Bergman 2001; Wells 2002; Wells and Nelson 1997). Biologists diagnose homologous structures (whether morphological or molecular) by first searching for characters or traits that are similar in form and position. Because not all features that are similar are necessarily inherited from a common ancestor, it is necessary to test structures before they can be declared homologous. Scientists conclude homology of structure only after comparing characters across many groups, looking for patterns of form, function, development, biochemistry, and presence and absence (Gishlick 2006). Common ancestry is not the rationale for homology but the explanation of the similarities (Padian 2005). This is why finding a pattern of similarities among organisms is not enough to establish homology. That pattern must show a genealogical relationship, and then it should be testable against observations in nature.

Third, sometimes it is quite easy to identify homologous structures, but at other times it is more difficult. The homology of leaves is easy, if you are considering only the familiar leaves of deciduous trees. But what about plants like cacti that don't appear to have leaves? In fact, the spines of a cactus, the red "flowers" (actually bracts) of a poinsettia, the pitcher of a pitcher plant, and the trap of a venus flytrap share their basic structure and development with leaves and therefore are considered homologous (Fig. 1; see http://evolution.berkeley. edu/evolibrary/article/homology_01).

Fourth, a structure can be homologous at one level (the bones that make up the wings of birds, pterosaurs, and

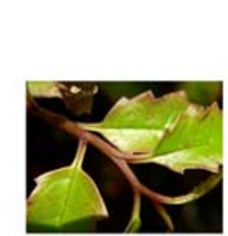

B.
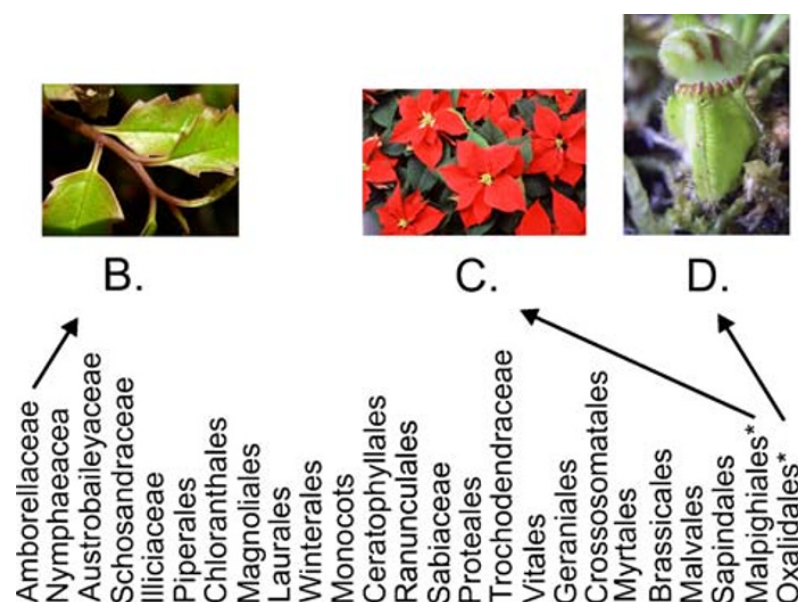

C.

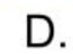

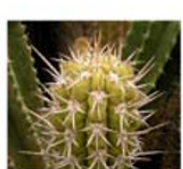

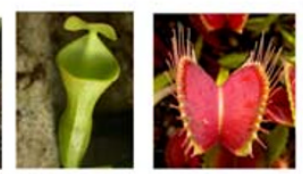

E.
$\mathrm{F}$.
G.

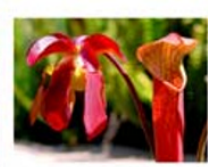

$\mathrm{H}$.

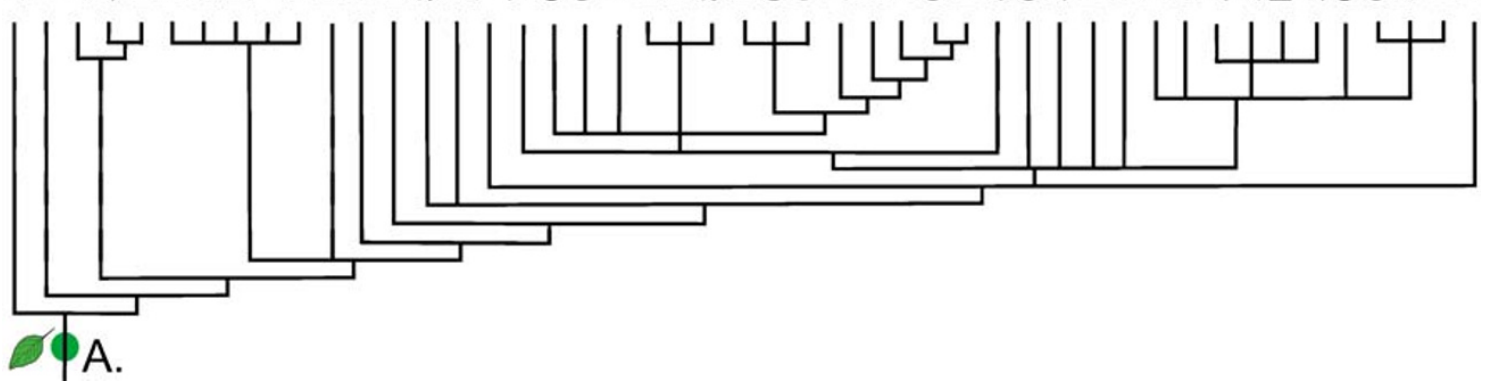

Fig. 1 Phylogeny for angiosperms showing clades (indicated with an asterisk) with modified leaves. a Cartoon representation of a leaf present in the ancestor; b Amborella trichipoda, an ancient lineage showing a simple leaf pattern; c Euphorbia pulcherrima; d. Cephalotus follicularis (Denis Barthel 2005); e. Armatocereus matucanensis (Frank Vincentz 2007); f. Nepenthes campanulata; g.Sarracenia rubra (Noeah Elhardt) 
bats), but not at another level (the wings in these three vertebrate flyers emerged independently from the forelimbs of non-flying ancestors). Similarly, the RNAse1 gene of ruminant artiodactyls and colobine monkeys is homologous in that the RNAsel gene is present in the common ancestor, but the gene was duplicated independently in each group (Fig. 2), and each lineage has subsequently evolved a new, yet parallel, function for this gene, without sharing the same pattern of mutation and amino acid substitution (Zhang 2003). Hence, homology is not simply a matter of the final form of an organism. Homology can exist at many levels: it can be genetic, histological, developmental, molecular, or behavioral, (see examples at http://evolution.berkeley.edu/evolibrary/article/0_0_0/ homology_04), and the underlying molecular, genetic, and developmental patterns are often complex. Varying and discordant patterns of homology across levels of organization should be expected and not viewed as evidence against evolution (Mindell and Meyer 2001).

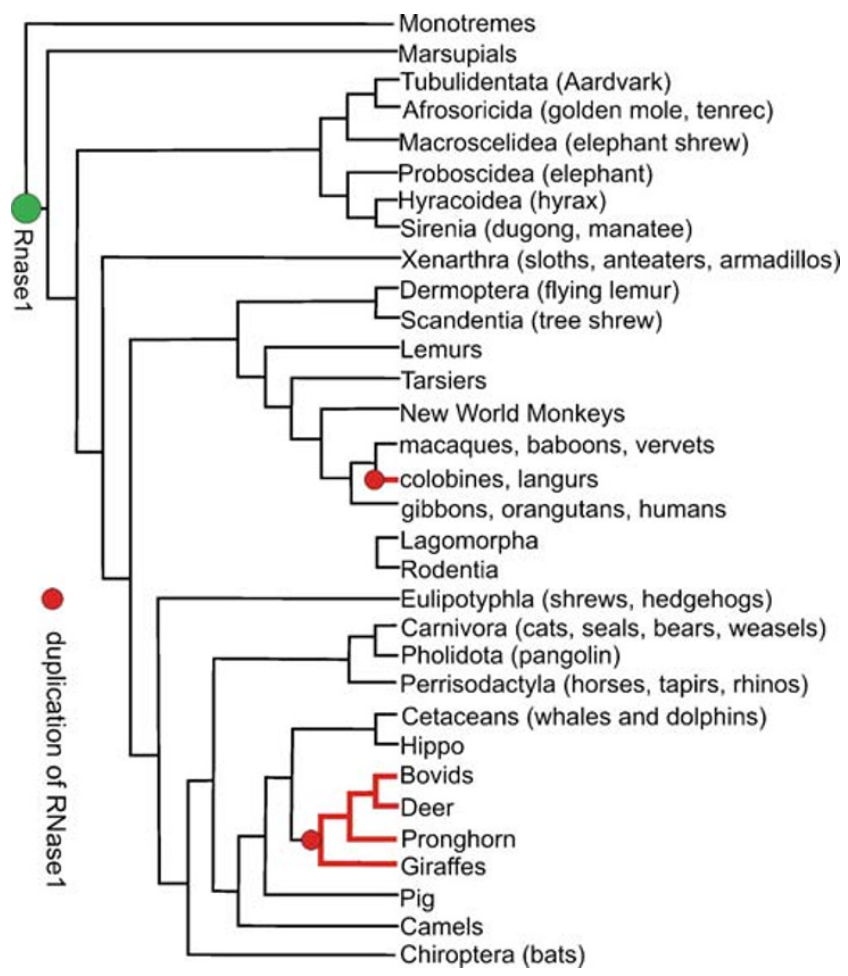

Fig. 2 Foregut fermentation has independently evolved twice in mammals, first in Rumantia, a lineage of cetartiodactlys that includes cow, sheep, moose, and giraffe - and then again in colobus, langur, and proboscis monkeys. The appearance of this mode of digestion has been accompanied by the independent recruitment of lysozymes as a bacteriolytic enzyme in the stomach and gene duplication of pancreatic RNase which is secreted by the pancreas RNasel gene in homologous, but the subsequent gene duplication and functional specialization occurred independently, but in parallel

\section{Value of Homology}

So how does homology help us understand the history and diversity of life? Hypotheses about homologous structures are more than just descriptive: they are also predictive, and these predictions can be falsified. Remembering that scientific classification reflects common evolutionary history, if we say that a whale is a mammal, we can examine its skeleton, nervous system, embryological development, hormones, and so on to establish that these features are derived from a common mammalian ancestor, that the unique features of whales are shared by descent only within the whales and their biological relatives, and that the overall external similarities with fishes (e.g., a streamlined body) appear as the ancestors of whales depended more and more on exploiting watery habitats. We can predict that there will be a series of changes that show the transition from land to deep oceans in ancestral whales and that there will be morphological, developmental, and biochemical homologies that link modern whales to the extant terrestrial descendants of their common ancestors (Peterson et al. 2007). For example, protein and mitochondrial DNA sequence data suggest whales and dolphins as most closely related to hippopatomid artiodactyls (Graur and Higgins 1994; Irwin and Arnason 1994; Gatesy et al. 1996; Gatesy 1997; Gatesy and O'Leary 2001; Murphy et al. 2001). Unique retroposon insertion events are also shared by cetaceans and hippos (Nikaido et al. 1999). The subsequent discovery of a stem-cetacean with doubletrochleated astragli, a uniquely derived characteristic of ancestral and extant artiodactylids (Gingerich et al. 2001; Thewissen et al. 2001) supported the predictive link between cetaceans and artiodactlylids made by the molecular data. Contrary to the claims of Wells and Nelson (1997), investigating homology is not engaging in a circular argument, but a dynamic process that leads to productive scientific research.

\section{Learning Homology}

Is homology too complex for $\mathrm{K}-12$ students to grasp? Contemporary child development research (for example, Poirel et al. 2008) makes it clear that by the middle school years, children are able to recognize and to assign objects to hierarchical categories with about the same accuracy as adults. So our goals for using biological homology to relate specific organisms with specific traits to branches on the tree of life based on their relationships with common ancestors can quite reasonably be accomplished in a typical $\mathrm{K}-12$ curriculum. The National Science Education Standards (NSES; NRC 1996) clearly identify the role of homology in addressing the evolution- 
ary basis of biological classification for high-school courses:

Biological classifications are based on how organisms are related. Organisms are classified into a hierarchy of groups and subgroups based on similarities which reflect their evolutionary relationships. (p. 185)

At the middle school level, the NSES point out the different levels at which we can assess homology: "Although different species might look dissimilar, the unity among organisms becomes apparent from an analysis of internal structures, the similarity of their chemical processes, and the evidence of common ancestry." (NRC 1996; p. 158)

For students to understand how homology can provide useful information, it is important to design lessons that bridge the gap from the folk model of "like goes with like" to one that shows the application of scientific inquiry. For example, in the lesson "Whale Ankles and DNA" on the Evolution and the Nature of Scientific Inquiry website (http://www.indiana.edu/ ensiweb/lessons/whale.ev.html), students see that homology exists on multiple levels and that the patterns of biological relationship among these levels are concordant.

What is more critical is that students learn to extract from the patterns of similarities and differences among organisms those features that are homologous from those that are not- to identify those characters that inform our understanding of evolutionary relationships. One approach is to go beyond dichotomous keys (for example, Michael McDarby has created an interactive classification key online at http://faculty.fmcc.suny.edu/mcdarby/ Pages/ClassificationKey/ClassificationIntro.htm) by having students use these keys to construct tree diagrams, depicting each decision node as a branching point on the tree. It will become clear quickly that some of the criteria used in these keys form genealogical patterns indicating common descent, while others do not. Learning to distinguish among these features with respect to homology allows students to demonstrate clearly their level of understanding of the nested hierarchy of evolutionary relationships.

Finally, hypotheses of homology must be made explicit to students through the choice of educational materials and activities. Homology is already in the textbooks, but there's a lot of room for improvement: textbooks could stand to be clearer on the definition of homology versus diagnosis, the descriptive and predictive nature of hypotheses, and the fact that hypotheses of homology are not just a matter of overall similarity of gross morphology, but emerge from development, biochemistry, genetics, and ecology. Educational materials must show exactly how homologous structures map onto phylogenies and are the result of common ancestry. A figure showing the bones of the forelimbs of various vertebrates (e.g., whale, bird, bat, salamander, crocodile, human, and mole) appears in most high school biology textbooks. In some cases, homologous bones are colorcoded; in other cases, they are not. Rarely are these structures mapped onto a phylogeny with the basic pattern shown in the common ancestor, as is depicted in an evogram (see Mead 2009 for further discussion of evograms and Zimmer 2009 for extensive examples). Organisms are never classified biologically by a single criterion or a single feature, but on the basis of mutually reinforcing and concordant patterns at multiple levels of analysis - and this is the essential concept for teaching homology in biology classes.

\section{References}

Begossi A, Clauzet M, Figueiredo JL, Garuana L, Lima RV, Lopes PF, et al. Are biological species and higher-ranking categories real? Fish folk taxonomy on Brazil's Atlantic forest coast and in the Amazon. Curr Anthropol. 2008;49(2):291-306.

Bergman J. Does homology provide evidence of evolutionary naturalism? (2001) TJ 15:26-33. http://wwwanswersingenesi sorg/tj/v15/i1/homologyasp. Accessed 28 Aug 2009

Berndt C. Biblical classification of life: a framework and reference for authentic biblical biology. Filer (ID): Elihu Publishing; 2000 .

Gatesy J. More DNA support for a Cetacea Hippopotamidae clade: the blood-clotting protein gene gamma-fibrinogen. Mol Biol Evol. 1997; 14:537-43.

Gatesy J, O'Leary MA. Deciphering whale origins with molecules and fossils. Trends Ecol Evol. 2001;16:562-70.

Gatesy J, Hayashi C, Cronin MA, Arctander P. Evidence from milk casein genes that cetaceans are close relatives of hippopotamid artiodactyls. Mol Biol Evol. 1996;13:954-63.

Gingerich PD, Ul Haq M, Zalmout IS, Khan IH, Malkani MS. Origin of whales from early artiodactyls: hands and feet of Eocene Protocetidae from Pakistan. Science. 2001;293:2239-42.

Gishlick A. Icon 3-Homology. National Center for Science Education. Available at http://ncseweb.org/creationism/analysis/icon-3homology. 2006.

Graur D, Higgins DG. Molecular evidence for the inclusion of cetaceans within the order Artiodactla. Mol Biol Evol. 1994;11:357-64.

Irwin DM, Árnason U. Cytochrome $b$ gene of marine mammals: phylogeny and evolution. J Mamm Evol. 1994;2(1):37-55.

Mead L. Transforming our thinking about transitional forms. Evolution: Education and Outreach. 2009;2:310-4.

Mindell D, Meyer A. Homology evolving. Trends Ecol Evol. 2001;16:434-40.

Murphy WJ, Eizirik E, O’Brien SJ, Madsen O, Scally M, Douady CJ, et al. Resolution of the early placental mammal radiation using Bayesian phylogenetics. Science. 2001;294:2348-51.

Nikaido M, Rooney AP, Okada N. Phylogenetic relationships among cetartiodactyls based on insertions of short and long interspersed elements: hippopotamuses are the closest extant relatives of whales. Proc Natl Acad Sci. 1999;96:10261-6. 
Nickels MN, Nelson CE. Beware of nuts and bolts: putting evolution back into the teaching of classification. Am Biol Teach. 2005;67:289-95.

NRC (National Research Council) National Science Education Standards Washington (DC): National Academy Press 1996

Padian K. Direct examination: creationist misrepresentation of homology and analogy. National Center for Science Education 2005. Available at: http://ncseweb.org/creationism/legal/directexamination-creationist-misrepresentations-homology-a.

Peterson KJ, Summons RE, Donoghue PCJ. Molecular palaeobiology. Paleontology. 2007;50:775-809.

Petto AJ, Meyers SC. Book Review of Biblical Classification of Life: A Framework and Reference for Authentic Biblical Biology, Berndt C. Reports of the National Center for Science Education 2004; 24(3-4): 40-1.

Poirel N, Mellet E, Houdé O, Pineau A. First came the tree, then the forest: developmental changes during childhood in the processing of visual local-global patterns according to the meaningfulness of the stimuli. Dev Psychol. 2008;44:245-53.

Thanukos A. Bringing homologies into focus. Evolution: education and outreach. 2008;1:498-504.

Thewissen JGM, Roe LJ, Hussain ST. Skeletons of terrestrial cetaceans and the relationship of whales to artiodactyls. Nature. 2001;413:277-81.

Wells J. Icons of evolution:science of myth? Why much of what we teach about evolution is wrong. Washington (DC): Regnery; 2002.

Wells J, Nelson P (1997) Homology: a concept in crisis. Origins and Design 18 (2) Available on line at http://www.arn.org/docs/ odesign/od182/hobi182.htm. Accessed 28 Aug 2009.

Zhang J. Parallel functional changes in the digestive RNases of ruminants and colobines by divergence amino acid substitutions. Mol Biol Evol. 2003;20:1310-7.

Zimmer C. The tangled bank: an introduction to evolution. Greenwood Village, Colorado: Roberts and Co. Publishing; 2009. 